\title{
Additional aspects of the generalized linear-fractional branching process
}

\author{
Nicolas Grosjean, Thierry Huillet \\ Laboratoire de Physique Théorique et Modélisation, \\ CNRS-UMR 8089 et Université de Cergy-Pontoise, \\ 2 Avenue Adolphe Chauvin, 95302, Cergy-Pontoise, FRANCE \\ E-mail: Nicolas.Grosjean@u-cergy.fr, Thierry.Huillet@u-cergy.fr
}

\begin{abstract}
We derive some additional results on the Bienyamé-Galton-Watson branching process with $\theta$-linear fractional branching mechanism, as studied in [16]. This includes: the explicit expression of the limit laws in both the sub-critical cases and the super-critical cases with finite mean, the long-run behavior of the population size in the critical case, limit laws in the super-critical cases with infinite mean when the $\theta$-process is either regular or explosive, results regarding the time to absorption, an expression of the probability law of the $\theta$-branching mechanism involving Bell polynomials, the explicit computation of the stochastic transition matrix of the $\theta$-process, together with its powers.
\end{abstract}

Keywords: Bienyamé-Galton-Watson branching process, $\theta$-linear fractional branching mechanism, population growth, Yaglom limits, powers of probability transition matrix.

\section{Introduction}

Recently, in [16, a family of branching mechanisms involving explosions was introduced: the so-called $\theta$-linear fractional family. It fixes the reproduction law of some specific Bienyamé-Galton-Watson branching processes [7, and it is given in terms of its probability generating function (pgf). This pgf family has the remarkable invariance under iterated composition property so that in principle the law of the population size at each generation can be computed. This family extends the classical linear-fractional model (obtained when $\theta=1$ ) whose study dates back to Schröder, (7], p. 9 and [17). This makes computation of important statistical quantities of great interest quite explicit. In this construction $\theta \in[-1,1]$, with very special properties for the cases $\theta \in\{-1,0,1\}$ when $\theta$ is an integer. We shall revisit this $\theta$-family and give some additional results, among which:

- the expression of the limit laws in the subcritical cases and super-critical cases with finite mean, solving respectively the associated Schröder and Poincaré functional equations.

- the long-run behavior of the population size in the critical case.

- limit laws in the super-critical cases with infinite mean when either the $\theta$-process is regular or explosive.

- information on the time to absorption defined as the infimum of the times to extinction and explosion. 
- an expression of the probability mass distribution of the $\theta$-branching mechanism, alternative to the one given in Proposition 4 of [16], using of Faa di Bruno formulae and Bell polynomials.

- the explicit computation of the stochastic transition matrix of the associated Bienyamé-Galton-Watson $\theta$-branching processes, together with its powers. This gives some access to the resolvent of such processes as a key ingredient to compute passage time statistics, hitting probabilities,...

We end up this work by a short section of examples where the following problem of concrete interest is addressed: what is the probability that, given the $\theta$-branching process has not yet gone extinct at some given generation, its extinction time be infinite with a large probability close to 1 . We do some computations in the special cases $\theta \in\{-1,0,1\}$.

\section{Generalities on Bienyamé-Galton-Watson (BGW) branch- ing processes}

We start with generalities on such BGW processes, including the case displaying finite-time explosion, [16].

\subsection{The pgf approach}

Consider a discrete-time Bienyamé-Galton-Watson branching process [7] whose reproduction law is given by the (sub-)probability law $\mathbf{P}(M=m)=: \pi(m), m \geq 0$ for the number $M$ of offspring per capita. We assume $\pi(0)>0$ so that the process can go extinct. We let $\phi(z)=\mathbf{E}\left(z^{M}\right)=\sum_{m \geq 0} \pi(m) z^{m}$ be the probability generating function of $M$ and we assume $\phi(1) \leq 1$.

With $N_{n}(1)$ the number of individuals alive at generation $n$ given $N_{0}=1$, we have

$$
\mathbf{E}\left(z^{N_{n}(1)}\right):=\mathbf{E}\left(z^{N_{n}} \mid N_{0}=1\right)=\phi^{\circ n}(z)
$$

where $\phi^{\circ n}(z)$ is the $n$-th composition of $\phi(z)$ with itself, 11. Similarly, if $N_{n}(i)$ is the number of individuals alive at generation $n$ given there are $N_{0}=i$ independent founders, we clearly get

$$
\mathbf{E}\left(z^{N_{n}(i)}\right):=\mathbf{E}\left(z^{N_{n}} \mid N_{0}=i\right)=\phi^{\circ n}(z)^{i} .
$$

We shall also let

$$
\tau_{i, j}=\inf \left(n \geq 1: N_{n}=j \mid N_{0}=i\right),
$$

the first hitting time of state $j \neq i$ given $N_{0}=i \neq 0$.

- If $\phi(1)<1$, there is a positive probability $1-\phi(1)=: \pi(\infty)$ that $M=\infty$ (explosion is made possible even at the first branching step): following [16, we shall speak of an explosive or non-regular process.

- If $\phi(1)=1$ (regular case), depending on $\mu:=\mathbf{E}(M) \leq 1$ (i.e. the (sub-)critical case ) or $\mu>1$ (supercritical case): the process $N_{n}$ (1) goes extinct with probability 1 or goes extinct with probability $\rho<1$ where $\rho$ is the smallest fixed point solution in $[0,1]$ to $\phi(\rho)=\rho$, respectively. In the latter case, the distribution of the time to extinction $\tau_{1,0}$ is given by

$$
\mathbf{P}\left(\tau_{1,0} \leq n\right)=\mathbf{P}\left(N_{n}(1)=0\right)=\phi^{\circ n}(0),
$$

\footnotetext{
${ }^{1}$ Throughout this work, a pgf will therefore be a function $\phi$ which is absolutely monotone on $(0,1)$ with all nonnegative derivatives of any order there, obeying $\phi(1) \leq 1$.
} 
and the process explodes with probability $\bar{\rho}:=1-\rho$, but not in finite time: only state $\{0\}$ is absorbing. Clearly also, if there are $i$ independent founders instead of simply 1 ,

$$
\mathbf{P}\left(\tau_{i, 0} \leq n\right)=\mathbf{P}\left(N_{n}(i)=0\right)=\phi^{\circ n}(0)^{i}
$$

- If $\phi(1)<1$ (explosive case), $\mu:=\mathbf{E}(M)=\infty$ because there is a positive probability $1-\phi(1)$ that $M=\infty$. Notice that

$$
\phi^{\prime}(1)=\mathbf{E}\left(M \cdot \mathbf{1}_{\{M<\infty\}}\right)=\sum_{m \geq 1} m \pi(m),
$$

if this quantity exists (is finite). If $\phi(1)<1$, state $\{\infty\}$ should be added to the state-space $\mathbb{N}_{0}=\{0,1, \ldots\}$ of $N_{n}(i)$ and then both states are $\{0, \infty\}$ are absorbing. In this supercritical case, $\rho<1$ always, and both the time to extinction $\tau_{1,0}$ and the time to explosion $\tau_{1, \infty}$ of $N_{n}(1)$ are finite with positive probability, now with

$$
\left\{\begin{array}{c}
\mathbf{P}\left(\tau_{1,0} \leq n\right)=\mathbf{P}\left(N_{n}(1)=0\right)=\phi^{\circ n}(0) \underset{n \rightarrow \infty}{\rightarrow} \rho=\mathbf{P}\left(\tau_{1,0}<\infty\right) \\
\mathbf{P}\left(\tau_{1, \infty}>n\right)=\mathbf{P}\left(N_{n}(1)<\infty\right)=\phi^{\circ n}(1) \underset{n \rightarrow \infty}{\rightarrow} \rho=\mathbf{P}\left(\tau_{1, \infty}=\infty\right) .
\end{array}\right.
$$

Thus $\rho$ and $\bar{\rho}$ are now also the probabilities that $\tau_{1,0}<\infty$ and $\tau_{1, \infty}<\infty$, respectively. We thus have

$$
\left\{\begin{array}{c}
\mathbf{P}\left(n<\tau_{1,0}<\infty\right)=\rho-\phi^{o n}(0) \\
\mathbf{P}\left(n<\tau_{1, \infty}<\infty\right)=\bar{\rho}-\left(1-\phi^{o n}(1)\right)=\phi^{o n}(1)-\rho, \text { and } \\
\mathbf{P}\left(n<\tau_{1}<\infty\right)=\mathbf{P}\left(0<N_{n}(1)<\infty\right)=\phi^{\text {on }}(1)-\phi^{\circ n}(0)
\end{array}\right.
$$

where we defined the global absorption time $\tau_{1}:=\tau_{1,0} \wedge \tau_{1, \infty}$. Clearly also, with $\tau_{i}:=\tau_{i, 0} \wedge \tau_{i, \infty}$

$$
\left\{\begin{array}{c}
\mathbf{P}\left(n<\tau_{i, 0}<\infty\right)=\rho^{i}-\phi^{\circ n}(0)^{i} \\
\mathbf{P}\left(n<\tau_{i, \infty}<\infty\right)=\left(1-\rho^{i}\right)-\left(1-\phi^{\circ n}(1)^{i}\right)=\phi^{o n}(1)^{i}-\rho^{i}, \text { and } \\
\mathbf{P}\left(n<\tau_{i}<\infty\right)=\mathbf{P}\left(0<N_{n}(i)<\infty\right)=\phi^{o n}(1)^{i}-\phi^{o n}(0)^{i}
\end{array}\right.
$$

Suppose a supercritical situation for which the extinction probability of $N_{n}(i)$ is smaller than 1 (always the case if $\phi(1)<1$ ). Of concrete interest is then the probability that, given the process $N_{n}(i)$ has not yet gone extinct at generation $n$, the extinction time of the process will be finite, namely

$$
\mathbf{P}\left(\tau_{i, 0}<\infty \mid N_{n}(i)>0\right)=\mathbf{P}\left(\tau_{i, 0}<\infty \mid \tau_{i, 0}>n\right) .
$$

We get

$$
\mathbf{P}\left(\tau_{i, 0}<\infty \mid N_{n}(i)>0\right)=\frac{\rho^{i}-\phi^{o n}(0)^{i}}{1-\phi^{o n}(0)^{i}},
$$

and the larger $n$, the smaller this probability because $\phi^{\circ n+1}(0)>\phi^{\circ n}(0)$. There is thus a value $n_{c}$ of $n$ for which, with probability $c$ close to 1 ,

$$
1-\mathbf{P}\left(\tau_{i, 0}<\infty \mid N_{n_{c}}(i)>0\right)=\frac{1-\rho^{i}}{1-\phi^{o n_{c}}(0)^{i}}=c(=\operatorname{say} 0.99) .
$$

This is the probability that some population with $i$ founders, still alive at generation $n_{c}$, will never go extinct. 


\subsection{The transition matrix approach}

A Bienaymé-Galton-Watson process is a time-homogeneous Markov chain with denumerable state-space $\mathbb{N}_{0}:=\{0,1, \ldots\}$. Its stochastic transition matrix is $P$, with entries $P(i, j)=\left[z^{j}\right] \phi(z)^{i}=\mathbf{P}\left(N_{1}(i)=j\right)$ (with $\left[z^{j}\right] \phi(z)^{i}$ meaning the $z^{j}$-coefficient of the $\left.\operatorname{pgf} \phi(z)^{i}\right)$. When there is explosion and in the supercritical cases, an interesting problem arises when conditioning $N_{n}$ either on extinction or on explosion. This may be understood as follows:

The harmonic column vector $\mathbf{h}$, solution to $P \mathbf{h}=\mathbf{h}$, is given by its coordinates $h(i)=\rho^{i}, i \geq 0$, because $\sum_{j \geq 0} P(i, j) \rho^{j}=\phi(\rho)^{i}=\rho^{i}$. Letting $D_{\mathbf{h}}:=\operatorname{diag}(h(0), h(1), \ldots)$, introduce the stochastic matrix $P_{\mathbf{h}}$ given by a Doob transform ([14] and ([15], p. 327): $P_{\mathbf{h}}=D_{\mathbf{h}}^{-1} P D_{\mathbf{h}}$ or $P_{\mathbf{h}}(i, j)=h(i)^{-1} P(i, j) h(j)=P(i, j) \rho^{j-i}, i, j \geq 0$. Note $h\left(N_{n}(i)\right)=\rho^{N_{n}(i)}$ is a martingale because $\mathbf{E}\left(h\left(N_{n}(i)\right)\right)=\phi^{\circ n}(\rho)^{i}=\rho^{i}=h(i)=$ $h\left(N_{0}(i)\right)$. Then $P_{\mathbf{h}}$ is the transition matrix of $N_{n}$ conditioned on almost sure extinction. Equivalently, when conditioning $N_{n}$ on almost sure extinction, one is led to a regular subcritical BGW process with new branching mechanism $\phi_{0}(z)=\phi(\rho z) / \rho$, satisfying $\phi_{0}(1)=1$ and $\phi_{0}^{\prime}(1)=\phi^{\prime}(\rho)<1$. Indeed, $\phi_{0}(z)=\sum_{j \geq 0} P_{\mathbf{h}}(1, j) z^{j}$. Upon iterating, we get $\phi_{0}^{\circ n}(z)=\phi^{\circ n}(\rho z) / \rho$.

Similarly, when conditioning $N_{n}$ on almost sure explosion, one is led to an explosive supercritical BGW process with new Harris-Sevastyanov branching mechanism $\phi_{\infty}(z)=[\phi(\rho+\bar{\rho} z)-\rho] / \bar{\rho}$, satisfying $\phi_{\infty}(0)=0$ and $\phi_{\infty}(1)=(\phi(1)-\rho) / \bar{\rho}<1$. Upon iterating, we have $\phi_{\infty}^{\circ n}(z)=\left[\phi^{\circ n}(\rho+\bar{\rho} z)-\rho\right] / \bar{\rho}$.

The second largest eigenvalue of $P$ is $\gamma=\phi^{\prime}(\rho)<1$. The corresponding eigenvector $\mathbf{u}$ obeys $P \mathbf{u}=\gamma \mathbf{u}$ with $u(i)=i \rho^{i-1}, i \geq 1$, because $\sum_{j \geq 1} P(i, j) j \rho^{j-1}=$

$\phi^{\prime}(\rho) i \phi(\rho)^{i-1}=\gamma i \rho^{i-1}$. Conditioning $N_{n}$ on never hitting $\{0, \infty\}$ in the remote future is given by the $Q$-process with stochastic transition matrix $Q=\gamma^{-1} D_{\mathbf{u}}^{-1} P D_{\mathbf{u}}$ or $Q(i, j)=\gamma^{-1} u(i)^{-1} P(i, j) u(j)=\gamma^{-1} \rho^{j-i} i^{-1} P(i, j) j, i, j \geq 1$ (see [12] and [16, Section 6 in the $\theta$-special case).

There are classes of discrete branching processes for which the pgf $\phi^{\circ n}(z)$ of $N_{n}(1)$ is exactly computable, thereby making the above computations concrete and somehow explicit.

\section{The $\theta$-linear fractional branching mechanism model, [16]}

With $|\theta| \leq 1, a, b>0$ and $z_{c} \geq 1$, we shall consider the $\theta$-linear fractional branching mechanism model, namely,

$$
\left\{\begin{array}{c}
\phi(z)=z_{c}-\left(a\left(z_{c}-z\right)^{-\theta}+b\right)^{-1 / \theta} \text { or } \\
\left(z_{c}-\phi(z)\right)^{-\theta}=a\left(z_{c}-z\right)^{-\theta}+b
\end{array}\right.
$$

and for those values of $z_{c} \geq 1$ and $a, b>0$ for which $\phi$ is a pgf with $\phi(1) \leq 1$. The case $\theta=0$ will be considered in (11).

\subsection{The boundary cases $\theta= \pm 1$}

The boundary cases $\theta= \pm 1$ deserve a special treatment that we shall first evacuate. 
- When $\theta=1, \phi(z)=z_{c}-\left(a\left(z_{c}-z\right)^{-1}+b\right)^{-1}$ is an homographic map. Assuming $a+b>1$ and introducing the probabilities $p_{0}=1 /(a+b), q=a /(a+b)$, with $p_{0}+q_{0}=1$ and $p+q=1$, this is also $\left(a=q / p_{0}, b=p / p_{0}\right)$

$$
\frac{1}{z_{c}-\phi(z)}=\frac{q}{p_{0}} \frac{1}{z_{c}-z}+\frac{p}{p_{0}}
$$

Note $\phi\left(z_{c}\right)=z_{c}$ but $z_{c}$ is not the convergence radius of $\phi$, which is $z_{c}+q / p$.

- In the particular case $z_{c}=1$, we have the two following interpretations for $\phi(z)$ :

Proposition $1(i)$ When $z_{c}=1, \phi(z)=q_{0}+p_{0}(q z) /(1-p z)$, the classical form of the simple linear fractional model. This pgf is the one of a random variable $M$ obtained as $M \stackrel{d}{=} G \cdot B$ (equality in law), where $B$ is Bernoulli( $\left.p_{0}\right)$ distributed, independent of $G$, a geometric $(1 / q)$ distributed random variable.

(ii) When $z_{c}=1$ and if $b<1$, we also have

$$
\phi(z)=\frac{1+(1-z) \frac{b-1}{a}}{1+(1-z) \frac{b}{a}},
$$

which can be put in the alternative form

$$
\phi(z)=\frac{\beta\left(\beta_{0}+\alpha_{0} z\right)}{1-\alpha\left(\beta_{0}+\alpha_{0} z\right)},
$$

while defining the probabilities $\alpha_{0}=(1-b) / a, \alpha=b$ and $\beta_{0}=1-\alpha_{0}, \beta=1-\alpha$. This $\phi(z)$ is thus the pgf of the random variable

$$
M \stackrel{d}{=} \sum_{k=1}^{G} B_{k},
$$

where $G$ now is geometric $(1 / \beta)$ distributed, independent of the sequence of independent and identically distributed $\left(B_{k}\right)_{k>1}$, with $B_{1}$ Bernoulli $\left(\alpha_{0}\right)$ distributed. $M$ is thus a Bernoulli-thinned version of $G$ in the sense of [18].

We have $\mu:=\mathbf{E}(M)=\phi^{\prime}(1)=p_{0} / q=1 / a$ and

$$
\left\{\begin{array}{c}
\phi^{\circ n}(z)=1-\left(a_{n}(1-z)^{-1}+b_{n}\right)^{-1} \text { where } \\
a_{n}=a^{n} \text { and } b_{n}=b\left(1+a+\ldots+a^{n-1}\right)
\end{array} .\right.
$$

Depending on $a>1, a=1$ or $a<1$, the corresponding branching process is subcritical, critical or supercritical. In the supercritical case $a=q / p_{0}<1$ the extinction probability is $\rho=q_{0} / p<1$.

- If now $z_{c}>1$, the additional constraints $\phi(0) \in(0,1)$ and $\phi(1) \leq 1$ impose $p_{0}<q+p z_{c} \leq p+p_{0}$. This family is of interest because its $n$-th iterate is explicit, also homographic, with

$$
\left\{\begin{array}{c}
\phi^{o n}(z)=z_{c}-\left(a_{n}\left(z_{c}-z\right)^{-1}+b_{n}\right)^{-1} \text { where } \\
a_{n}=a^{n} \text { and } b_{n}=b\left(1+a+\ldots+a^{n-1}\right) .
\end{array}\right.
$$


Thus for instance, if $z_{c}>1$, and $q+p z_{c}<p+p_{0}$

$$
\mathbf{P}\left(n<\tau_{1}<\infty\right)=\phi^{\circ n}(1)-\phi^{\circ n}(0)=\frac{a_{n}}{\left(a_{n}+b_{n} z_{c}\right)\left(a_{n}+b_{n}\left(z_{c}-1\right)\right)},
$$

with

$$
\mathbf{P}\left(n<\tau_{1}<\infty\right) \underset{n \rightarrow \infty}{\sim}\left\{\begin{array}{c}
\frac{(a-1)^{2}}{\left(a-1+b z_{c}\right)\left(a-1+b\left(z_{c}-1\right)\right)} a_{n}^{-1} \text { if } a>1 \\
\frac{a-1)^{2}}{b^{2} z_{c}\left(z_{c}-1\right)} a^{n} \text { if } a<1 \\
\frac{1}{b^{2} z_{c}\left(z_{c}-1\right)} n^{-2} \text { if } a=1
\end{array} .\right.
$$

When $a=1\left(p_{0}=q\right.$ and $\left.q_{0}=p\right)$, the tails of $\tau_{1}$ are no longer asymptotically geometric, rather they are power-law with tail index 2 .

- When $\theta=-1, \phi(z)=a z+z_{c}(1-a)-b$ is the affine map and, if $\phi(1)=1$, the corresponding branching process is the regular death process as each individual can only either die or survive upon splitting. With $\pi(1)=a, \pi(0)=z_{c}(1-a)-b=$ $1-\pi(1), \phi(z)=\pi(1) z+\pi(0)$ and the corresponding branching process is subcritical, always, with mean $\mu=\pi(1)=a<1$. With $\pi_{n}(0)+\pi_{n}(1)=1$, we have

$$
\phi^{\circ n}(z)=\pi_{n}(0)+\pi_{n}(1) z, \text { where } \pi_{n}(1)=\pi(1)^{n} .
$$

If $\phi(1)<1$, the corresponding branching process is an explosive process where each individual can either die, survive or give birth to infinitely many descendants on splitting. The additional constraints $\phi(0) \in(0,1)$ and $\phi(1)<1$ impose $\pi(1)=$ $a \in(0,1), \pi(0)=z_{c}(1-a)-b<1-\pi(1)=1-a$, thus $\left(z_{c}-1\right)(1-a)<b$. This family is of interest because its $n$-th iterate is again explicit

$$
\left\{\begin{array}{c}
\phi^{o n}(z)=z_{c}-\left(a_{n}\left(z_{c}-z\right)+b_{n}\right) \text { with } \\
a_{n}=a^{n} \text { and } b_{n}=b\left(1+a+\ldots+a^{n-1}\right)=b \frac{1-a^{n}}{1-a}
\end{array}\right.
$$

and again in the same class of affine maps. With $\pi_{n}(0)+\pi_{n}(1)<1$, this is also

$$
\phi^{o n}(z)=\pi_{n}(0)+\pi_{n}(1) z, \text { where } \pi_{n}(0)=\pi(0) \frac{1-a^{n}}{1-a} \text { and } \pi_{n}(1)=\pi(1)^{n} .
$$

We have

$$
\begin{aligned}
& \mathbf{P}\left(N_{n}(1)<\infty\right)=\pi_{n}(0)+\pi_{n}(1)=\pi(0) \frac{1-a^{n}}{1-a}+\pi(1)^{n} \\
& \underset{n \rightarrow \infty}{\rightarrow} \mathbf{P}\left(N_{\infty}(1)<\infty\right):=\pi(0) /(1-a)<1 .
\end{aligned}
$$

$\mathbf{P}\left(N_{n}(1)=\infty\right)$ is an increasing sequence. The relative rate of approach of $\mathbf{P}\left(N_{n}(1)=\infty\right)$ to its limiting value decays geometrically with

$$
\frac{\mathbf{P}\left(N_{\infty}(1)=\infty\right)-\mathbf{P}\left(N_{n}(1)=\infty\right)}{\mathbf{P}\left(N_{\infty}(1)=\infty\right)}=a^{n} .
$$

Note $\mathbf{P}\left(n<\tau_{1}<\infty\right)=\phi^{o n}(1)-\phi^{\circ n}(0)=a^{n}$, an exact geometric distribution.

\subsection{The case $\theta \in(-1,1)$}

Although we deal here with the case $\theta \in(-1,1)$, we, somehow abusively, extend the range of the parameter set to its boundary whenever it causes no particular problem.

- With $\theta \in(-1,1), a, b>0$ and $z_{c}=\sup (z>0: \phi(z)<\infty) \geq 1$, let us reconsider $\phi(z)$ as defined by (11). Note now $\phi\left(z_{c}\right) \leq z_{c}\left(=z_{c}\right.$ if $\left.\theta \in(0,1]\right)$ and $z_{c}>1$ could 
produce $\phi(1)<1$, the explosion opportunity. This family is of interest because its $n$-th iterate is also explicit with (if $\theta \neq 0$ )

$$
\left\{\begin{array}{c}
\phi^{\circ n}(z)=z_{c}-\left(a_{n}\left(z_{c}-z\right)^{-\theta}+b_{n}\right)^{-1 / \theta} \text { where } \\
a_{n}=a^{n} \text { and } b_{n}=b\left(1+a+\ldots+a^{n-1}\right),
\end{array}\right.
$$

and it is in the same class as $\phi$, although for a different set of parameters $a, b$ (an invariance under iteration property).

The case $\theta=0$ is defined by continuity from the case $\theta \in(-1,1) \backslash\{0\}$ while observing

$\phi(z)=z_{c}-\left(a\left(z_{c}-z\right)^{-\theta}+(1-a)\left(z_{c}-\rho\right)^{-\theta}\right)^{-1 / \theta} \underset{|\theta| \rightarrow 0}{\rightarrow} z_{c}-\left(z_{c}-\rho\right)^{1-a}\left(z_{c}-z\right)^{a}$,

with $\phi(1)<1$ if $z_{c}>1$. Notice that if $z_{c}=1, \phi(1)=1$ and $\mu=\infty$ (the only regular case with infinite mean).

There are three cases, depending on $\mu:=\mathbf{E}(M)<1,=1$ or $>1$ :

- $(A)$ : subcritical cases:

(i) If $\theta \in(0,1], z_{c}=1, a>1, b>0$, then $\mu=a^{-1 / \theta}<1$. Again, if $\theta=1$, $\phi(z)=q_{0}+p_{0} q z /(1-p z)$ with $p_{0}=1 /(a+b), p=b /(a+b)$, the classical form of the 1-fractional model as the composition of a $\operatorname{Bernoulli}\left(p_{0}\right)$ pgf with the one of a geometric $(p / q)$ pgf.

(ii) If $\theta \in(-1,1], z_{c}>1, a \in(0,1)$ and $b=(1-a)\left(z_{c}-1\right)^{-\theta}$, then $\mu=a<1$.

(iii) If $\theta=-1, z_{c}=1$ and $a \in(0,1)$, then $\mu=a<1$.

- $(B)$ : critical case $(\mu=1)$ : this situation occurs only when $\theta \in(0,1], z_{c}=1$, $a=1, b>0$.

- $(C)$ : supercritical case $(\infty \geq \mu>1): \theta \in(-1,1], z_{c} \geq 1, a \in(0,1)$, $b=(1-a)\left(z_{c}-\rho\right)^{-\theta}$ where equivalently $\rho=z_{c}-((1-a) / b)^{1 / \theta}$ is the extinction probability of the process, as the smallest solution in the interval $[0,1]$ to $\phi(\rho)=\rho$ with $\rho \in(0,1)$. We have $a=\phi^{\prime}(\rho)$.

In the supercritical case with $z_{c}>1$, then $\mu=\infty$ because in this case,

$$
\phi(1)=z_{c}-\left(a\left(z_{c}-1\right)^{-\theta}+(1-a)\left(z_{c}-\rho\right)^{-\theta}\right)^{-1 / \theta}<1
$$

and $M=\infty$ with a positive probability.

In general, we have $\phi^{\prime}(1)=a\left(a+b\left(z_{c}-1\right)^{\theta}\right)^{-(\theta+1) / \theta}=a\left(a+(1-a)\left(\frac{z_{c}-1}{z_{c}-\rho}\right)^{\theta}\right)^{-(\theta+1) / \theta}$ which coincides with $\mu$ if $z_{c}=1$. We conclude that in the supercritical case with $z_{c}=1$

$$
\mu=\left\{\begin{array}{c}
\infty \text { if } \theta \in(-1,0], a \in(0,1) \\
a^{-1 / \theta} \text { if } \theta \in(0,1], a \in(0,1)
\end{array} .\right.
$$

In the first case,

- if $\theta \in(-1,0), a \in(0,1)$ then $\mu=\infty$ as a result of finite-time explosion because $\phi(1)=1-\left((1-a)(1-\rho)^{-\theta}\right)^{-1 / \theta}<1$ (explosive case).

- if $\theta=0, a \in(0,1), \mu=\infty$ even though $\phi(1)=1$ (the only regular case with infinite mean). 


\section{Remarks:}

(i) To the subset of models $(A)$ to $(B)$, we have added the special affine case $\theta=-1$ with $z_{c}=1$. If $z_{c}>1$, the affine model is supercritical with $\mu=\infty$ because the branching event $M=\infty$ has a positive probability. The special case $\theta=0$ is supercritical with $\mu=\infty$ both when $z_{c}=1$ and $z_{c}>1$. The special case $\theta=1$ corresponds to the standard linear fractional model and its criticality status has been included in the above classification.

(ii) Due to the invariance under iterated composition of the $\theta$-family of pgfs, it holds that $\left[\phi^{\circ n}\right]^{-1}(z)=\phi^{\circ(-n)}(z)$ : the inverse function of $\phi^{\circ n}(z)$ simply is $\phi^{\circ(-n)}(z)$, obtained while substituting $-n$ to $n$ in $\phi^{o n}(z)$, (a time-reversal property).

\section{Limit laws}

We shall investigate different limit laws concerning cases $(A)$ to $(C)$.

\subsection{Limit laws (subcritical/critical and super-critical with fi- nite mean cases)}

- Subcritical case with $\mu<1$ :

In the subcritical case, considering the population size, given it is positive, gives rise to a limiting random variable as the generation number goes to infinity. This limiting random variable is known as the quasi-stationary Yaglom limit, 20.

In our context, there are three different cases where this situation can occur:

$(A) /(i)$. In this case, with $\theta \in(0,1], z_{c}=1, a>1, b>0$ and $\phi(z)=1-$ $\left(a(1-z)^{-\theta}+b\right)^{-1 / \theta}, N_{n} \mid N_{n}>0 \stackrel{d}{\rightarrow} N_{\infty}$ where $N_{\infty}$ is a random variable with value in $\mathbb{N}_{0}=\{1,2, \ldots\}$ whose pgf $\phi_{\infty}(z):=\mathbf{E}\left(z^{N_{\infty}}\right)=\sum_{l \geq 1} \pi_{\infty}(l) z^{l}$ obeys the Schröder functional equation

$$
\bar{\phi}_{\infty}(\phi(z))=\mu \bar{\phi}_{\infty}(z), \bar{\phi}_{\infty}(z)=1-\phi_{\infty}(z), \mu=a^{-1 / \theta} .
$$

Note $\phi(z)=\bar{\phi}_{\infty}^{-1}\left(\mu \bar{\phi}_{\infty}(z)\right)$ and thus $\phi^{o n}(z)=\bar{\phi}_{\infty}^{-1}\left(\mu^{n} \bar{\phi}_{\infty}(z)\right)$.

Proposition 2 With $\alpha=\frac{a-1}{a+b-1}$ and $\beta=\frac{b}{a+b-1}(\alpha+\beta=1)$, we find the pgf of the Yaglom quasi-stationary limit $N_{\infty}$ as

$$
\phi_{\infty}(z)=1-\frac{1-z}{\left(\alpha+\beta(1-z)^{\theta}\right)^{1 / \theta}},
$$

obeying $\phi_{\infty}(0)=0, \phi_{\infty}(1)=1$ and with mean $\mu_{\infty}:=\phi_{\infty}^{\prime}(1)=\alpha^{-1 / \theta}=\left(\frac{a-1}{a+b-1}\right)^{-1 / \theta}$.

If in particular $\theta=1$,

$$
\phi_{\infty}(z)=\frac{z}{1+\frac{\beta}{\alpha}(1-z)}=\frac{\alpha z}{1-\beta z}
$$

is the pgf of a geometric random variable with mean $1+\beta / \alpha=1 / \alpha$. Thus $\pi_{\infty}(l)=$ $\mathbf{P}\left(N_{\infty}=l\right)=\alpha \beta^{l-1}, l \geq 1$, decays geometrically fast.

Corollary 3 Defining $\bar{\pi}_{\infty}(k):=\sum_{l>k} \pi_{\infty}(l)$,

$$
\bar{\pi}_{\infty}(k) \underset{k \uparrow \infty}{\sim}-\frac{1}{\Gamma(-\theta)} \frac{\beta}{\theta \alpha^{1+1 / \theta}} k^{-(1+\theta)},
$$


displaying power law tails with index $1+\theta$ if $\theta \in(0,1): N_{\infty}$ only has moments of order strictly less than $1+\theta$.

Proof: If $\theta \in(0,1)$, the tail pgf of $N_{\infty}$ is

$$
\frac{1-\phi_{\infty}(z)}{1-z}=\left(\alpha+\beta(1-z)^{\theta}\right)^{-1 / \theta},
$$

and the proof follows from Tauberian theorem, observing

$$
\left(\alpha+\beta(1-z)^{\theta}\right)^{-1 / \theta} \underset{z \downarrow 1}{\sim} \mu_{\infty}\left(1-\frac{\beta}{\alpha \theta}(1-z)^{\theta}\right) .
$$

$(A) /(i i)$. In the subcritical case $(A) /(i i)$, with $\theta \in(-1,1] \backslash\{0\}, z_{c}>1, a \in$ $(0,1), b=(1-a)\left(z_{c}-1\right)^{-\theta}$. Here, with $\phi(1)=1$ (a regular case)

$$
\phi(z)=z_{c}-\left(a\left(z_{c}-z\right)^{-\theta}+(1-a)\left(z_{c}-1\right)^{-\theta}\right)^{-1 / \theta} \text { and } \mu=\phi^{\prime}(1)=a<1 .
$$

Let $h(z)=z_{c}-z=h^{-1}(z), g(z)=z^{-\theta}$ and $f(z)=g(h(z))=\left(z_{c}-z\right)^{-\theta}$. The above equation is also 9

$$
f(\phi(z))=a f(z)+(1-a) f(1) .
$$

Let us look for an invertible function $A(z)$ with inverse $B(x)=A^{-1}(x)$ such that $\phi(z)=B(\mu A(z))=B(a A(z))$. Combining the two equations, we should have

$$
\begin{aligned}
f \circ B(a A(z)) & =a f(z)+(1-a) f(1) \text { or } \\
f \circ B(a x) & =a f \circ B(x)+(1-a) f(1)
\end{aligned}
$$

leading to an affine solution $f \circ B(x)=\alpha x+\beta$ with $\beta=f(1)$ and $\alpha$ left undetermined so far. We get

$$
\begin{aligned}
& B(x)=f^{-1}(\alpha x+f(1))=z_{c}-(\alpha x+f(1))^{-1 / \theta} \\
& A(z)=B^{-1}(z)=\frac{1}{\alpha}\left(\left(z_{c}-z\right)^{-\theta}-\left(z_{c}-1\right)^{-\theta}\right) .
\end{aligned}
$$

We thus have $\phi_{\infty}(z)=1-A(z)=1-\frac{1}{\alpha}\left(\left(z_{c}-z\right)^{-\theta}-\left(z_{c}-1\right)^{-\theta}\right)$ with $\phi_{\infty}(1)=1$. Imposing $\phi_{\infty}(0)=0$ yields $\alpha=z_{c}^{-\theta}-\left(z_{c}-1\right)^{-\theta}$ and so

\section{Proposition 4}

$$
\phi_{\infty}(z)=1-\left(\frac{\left(z_{c}-z\right)^{-\theta}-\left(z_{c}-1\right)^{-\theta}}{z_{c}^{-\theta}-\left(z_{c}-1\right)^{-\theta}}\right)=\frac{1-\left(1-z / z_{c}\right)^{-\theta}}{1-\left(1-1 / z_{c}\right)^{-\theta}}
$$

is the searched pgf of the unique Yaglom limit $N_{\infty}$ in this case study. It has finite mean $\phi_{\infty}^{\prime}$ (1) (and moments) and $\mathbf{P}\left(N_{\infty}=k\right)$ is asymptotically equivalent to $k^{\theta-1} z_{c}^{-k}$ with both power-law and geometrically decaying factors.

The case $\theta=0$ is finally obtained by continuity. 
Corollary 5 If $\theta=0$, we get a logarithmic pgf for $N_{\infty}$ as a result of

$$
\phi_{\infty}(z)=\frac{1-\left(1-z / z_{c}\right)^{-\theta}}{1-\left(1-1 / z_{c}\right)^{-\theta}} \underset{|\theta| \rightarrow 0}{\rightarrow} \frac{-\log \left(1-z / z_{c}\right)}{-\log \left(1-1 / z_{c}\right)},
$$

with mean $\phi_{\infty}^{\prime}(1)=-\frac{1}{\left(z_{c}-1\right) \log \left(1-1 / z_{c}\right)}>1$.

$(A) /($ iii $)$. In the subcritical case $(A) /($ iii $)$ (pure death case with $\phi(z)=\pi(0)+$ $\pi(1) z$ and $\mu=\pi(1)<1), N_{n} \mid N_{n}>0 \stackrel{d}{\rightarrow} N_{\infty}$ where simply $N_{\infty}=1$ whose pgf $\phi_{\infty}(z):=\mathbf{E}\left(z^{N_{\infty}}\right)=z$ clearly obeys the Schröder functional equation

$$
\bar{\phi}_{\infty}(\phi(z))=\mu \bar{\phi}_{\infty}(z), \bar{\phi}_{\infty}(z)=1-z .
$$

Obviously, $\phi(z)=\bar{\phi}_{\infty}^{-1}\left(\mu \bar{\phi}_{\infty}(z)\right)$ and thus $\phi^{\circ n}(z)=\bar{\phi}_{\infty}^{-1}\left(\mu^{n} \bar{\phi}_{\infty}(z)\right)=1-\mu^{n}(1-z)$ as required.

- Critical case with $\mu=1$ :

This concerns the case $(B)$ when $\theta \in(0,1], z_{c}=1, a=1, b>0$. We have

$$
\begin{gathered}
\phi(z)=1-\left((1-z)^{-\theta}+b\right)^{-1 / \theta} \\
\phi^{\circ n}(z)=1-\left((1-z)^{-\theta}+n b\right)^{-1 / \theta} .
\end{gathered}
$$

This is a regular case with $\phi(1)=1$.

Proposition 6 The process goes extinct with probability 1 but it takes a long time to do so. Indeed,

$$
\begin{aligned}
& \mathbf{P}\left(\tau_{1,0}>n\right)=1-\phi^{\circ n}(0)=(1+n b)^{-1 / \theta} \sim(n b)^{-1 / \theta}, \\
& \mathbf{P}\left(\tau_{i, 0}>n\right)=1-\phi^{\circ n}(0)^{i} \sim i(n b)^{-1 / \theta}, \text { for large } n,
\end{aligned}
$$

with persistent heavy tails, non-geometric.

The pgf of $N_{n}(1)$ conditioned on $N_{n}(1)>0$ is

$$
\frac{\phi^{o n}(z)-\phi^{o n}(0)}{1-\phi^{o n}(0)}
$$

therefore

$$
\begin{aligned}
\mathbf{E}\left(N_{n}(1) \mid N_{n}(1)>0\right) & =(1+n b)^{1 / \theta} \sim b^{1 / \theta} n^{1 / \theta}, \\
\mathbf{E}\left(N_{n}(i) \mid N_{n}(i)>0\right) & \sim i b^{1 / \theta} n^{1 / \theta}, \text { for large } n,
\end{aligned}
$$

with slow algebraic growth of order $n^{1 / \theta}$ in $n$. A direct computation shows that

$$
\phi^{\prime \prime}(z)=\frac{b(\theta+1)(1-z)^{\theta-1}}{\left(1+b(1-z)^{\theta}\right)^{1 / \theta+2}} .
$$


Because $\phi^{\prime \prime}(1)=2 b<\infty$ only when $\theta=1$, it holds ([7, [1]) that, if $\theta=1$, $\mathbf{E}\left(N_{n}(1) \mid N_{n}(1)>0\right) \sim n b$ and

$$
\mathbf{P}\left(\frac{N_{n}(1)}{n b}>x \mid N_{n}(1)>0\right) \underset{n \rightarrow \infty}{\rightarrow} e^{-x}, x>0 .
$$

- Regular supercritical case with $\mu<\infty$.

In the supercritical case $(C)$ for which $\mu=a^{-1 / \theta}<\infty\left(z_{c}=1, \theta \in(0,1]\right.$, $a \in(0,1)), \mu^{-n} N_{n} \stackrel{d}{\rightarrow} W$ where $W \geq 0$ is a random variable with value in $\mathbb{R}_{+}=[0, \infty)$ whose Laplace-Stieltjes transform $\phi_{W}(\lambda):=\mathbf{E}\left(e^{-\lambda W}\right), \lambda \geq 0$, obeys the Poincaré functional equation

$$
\phi_{W}(\mu \lambda)=\phi\left(\phi_{W}(\lambda)\right) .
$$

Note $\phi(z)=\phi_{W}\left(\mu \phi_{W}^{-1}(z)\right)$ and thus $\phi^{o n}(z)=\phi_{W}\left(\mu^{n} \phi_{W}^{-1}(z)\right)$.

Proposition 7 With $\alpha=\frac{1-a}{a+b-1}>0$ and $\beta=\frac{b}{a+b-1}>0(\beta-\alpha=1)$, if $z_{c}=1$, the Laplace-Stieltjes transform of the asymptotic growth rate $W$ of $\mu^{-n} N_{n}$ is

$$
\phi_{W}(\lambda)=1-\lambda \alpha^{1 / \theta}\left(\beta \lambda^{\theta}+1\right)^{-1 / \theta}=\rho+\bar{\rho}\left(1-\left(1+\beta^{-1} \lambda^{-\theta}\right)^{-1 / \theta}\right) .
$$

The extinction probability is $\phi_{W}(\infty)=\rho=1-\left(\frac{\alpha}{\beta}\right)^{1 / \theta}$ and $W$ has an atom at $r=0$ with mass $\rho$. We have $\phi_{W}(0)=1$ and the mean of $W$ is $\mu_{W}:=-\phi_{W}^{\prime}(0)=\alpha^{1 / \theta}$.

For general supercritical BGW processes, the limiting $W$ given $W>0$ is known to be infinitely divisible in some but not all cases [2]. We don't know if $W \mid W>0$ here in (18) is infinitely divisible or not.

Corollary 8 If $\theta=1$,

$$
\phi_{W}(\lambda)=1-\lambda \alpha(\beta \lambda+1)^{-1}=\frac{\lambda+1}{\beta \lambda+1}=\frac{1}{\beta}+\left(1-\frac{1}{\beta}\right) \frac{1}{1+\beta \lambda}
$$

is the Laplace-Stieltjes transform of an exponential random variable with an atom at 0 with mass $\rho=1 / \beta$ and mean $\bar{\rho} \beta=\frac{1-a}{a+b-1}=\alpha$. And $\mathbf{P}(W>r \mid W>0) \sim e^{-r / \beta}$ decays exponentially fast.

Furthermore, using [5], p. 445,

Corollary 9 If $\theta \in(0,1), \phi_{W}(\lambda) \sim \rho+\bar{\rho}\left(1-\beta^{1 / \theta} \lambda\right)$ as $\lambda$ is close to 0 , meaning exponential tails again, now with $\mathbf{P}(W>r \mid W>0) \underset{r \rightarrow \infty}{\sim} e^{-r / \beta^{1 / \theta}}$. As $\lambda$ is close to $\infty$, $\phi_{W}(\lambda) \sim \rho+\bar{\rho}(\beta \theta)^{-1} \lambda^{-\theta}$, meaning heavy algebraic left tails $\mathbf{P}(W \leq r \mid W>0) \underset{r \rightarrow 0}{\sim}$ $(\beta \theta)^{-1} r^{\theta} / \Gamma(1+\theta)$. 


\subsection{Limit laws (super-critical with infinite mean cases)}

There are two different regimes, depending on $\mu=\infty$ resulting or not from finite-time explosion:

- Regular case. If $z_{c}=1$, the infinite mean case $\mu=\infty$ occurs when $\theta=0$, $a \in(0,1)$. In such a case, $\phi(z)=1-(1-\rho)^{1-a}(1-z)^{a}$ and $\phi(1)=1$ (no finite-time explosion). With $E(1)$ a standard mean 1 exponential random variable

$$
a^{n} \log \left(1+N_{n}(1)\right) \stackrel{\text { a.s. }}{\rightarrow} W=\left\{\begin{array}{c}
0 \text { with probability } \rho \\
E(1) \text { with probability } \bar{\rho} \text {, as } n \rightarrow \infty
\end{array}\right.
$$

and conditionally given $N_{n}(1)$ does not go extinct, $N_{n}(1)$ grows at double exponential speed.

The pgf of $N_{n}(1)$ given explosion indeed is

$$
\phi_{\infty}^{\circ n}(z)=1-(1-z)^{a^{n}},
$$

and the above statement follows from the martingale proof of [8, proposition 3.8, adapted to the discrete time context. Similar regular models with infinite offspring mean were recently studied in $[10$.

Remark: It can be checked that, with $\log _{a} b=\log b / \log a$ and $A(z)=1-$ $\log _{1-\rho}(1-z), z<1$,

$$
\phi(z)=A^{-1}(a A(z)), \text { so that } \phi^{\circ n}(z)=A^{-1}\left(a^{n} A(z)\right) .
$$

This is an alternative way to see that such a branching model is 'integrable'.

- Explosive case. If $(i) z_{c}>1$ and $\theta \in(-1,1], a \in(0,1), b=(1-a)\left(z_{c}-\rho\right)^{-\theta}$ with $\rho \in(0,1)$ or $(i i)$ if $z_{c}=1$ and $\theta \in(-1,0), a \in(0,1)$ and $b=(1-a)(1-\rho)^{-\theta}$, where $\rho \in(0,1)$, then $N_{n}(1)$ can be infinite even in the first iteration step (finite time explosion). What only matters in this context is the time $\tau_{1, \infty}$ to explosion and also $\tau_{1}=\tau_{1,0} \wedge \tau_{1, \infty}$, as well as $\tau_{i}$. We get

Proposition 10 (i) When $\theta \in(-1,1], z_{c}>1, a \in(0,1), b=(1-a)\left(z_{c}-\rho\right)^{-\theta}$ with $\rho \in(0,1)$, leading to $\mu=\infty$, we have for instance

$$
\mathbf{P}\left(n<\tau_{1}<\infty\right)=\phi^{\circ n}(1)-\phi^{\circ n}(0) \underset{n \rightarrow \infty}{\sim}\left(\frac{1-a}{b}\right)^{1+1 / \theta}\left(\left(z_{c}-1\right)^{\theta}-z_{c}^{\theta}\right) a^{n},
$$

showing that $\tau_{1}$ is tail equivalent to a geometric random variable. Similarly

$$
\mathbf{P}\left(n<\tau_{i}<\infty\right)=\phi^{\circ n}(1)^{i}-\phi^{\circ n}(0)^{i} \underset{n \rightarrow \infty}{\sim} i\left(\frac{1-a}{b}\right)^{1+i / \theta}\left(\left(z_{c}-1\right)^{\theta}-z_{c}^{\theta}\right) a^{n} .
$$

(ii) If $z_{c}=1$ and $\theta \in(-1,0), a \in(0,1)$ and $b=(1-a)(1-\rho)^{-\theta}$, where $\rho \in(0,1)$, we have

$$
\mathbf{P}\left(n<\tau_{i}<\infty\right)=\phi^{\circ n}(1)^{i}-\phi^{\circ n}(0)^{i} \underset{n \rightarrow \infty}{\sim}-i \frac{(1-\rho)^{-(1+i / \theta)}}{\theta} a^{n},
$$

still with the tail equivalence to a geometric random variable. 


\section{Powers of the $\theta$-process transition matrix obtained by iter- ation}

So far we dealt with this $\theta$-family of pgfs for the reproduction law. It remains to compute the probability mass function to which they are associated. A related question is to compute the stochastic transition matrix of the $\theta$-branching processes together with its powers in time. We shall now address these points. We shall start with the cases $\theta \in(-1,1) \backslash\{0\}$ before addressing the special cases $\theta \in\{-1,0,1\}$.

\subsection{The case $\theta \in(-1,1) \backslash\{0\}$}

- We start with the reproduction law. Let $\phi(z)=z_{c}-\left(a\left(z_{c}-z\right)^{-\theta}+b\right)^{-1 / \theta}$ be a $\theta$-pgf with $\phi(z) \leq 1$. We first wish to compute the associate probability mass distribution: $\pi(k)=\left[z^{k}\right] \phi(z)$. Introduce $\phi_{c}(z):=z_{c}^{-1} \phi\left(z_{c} z\right)$, so with $\phi_{c}(z)=$ $1-\left(a(1-z)^{-\theta}+b z_{c}^{\theta}\right)^{-1 / \theta}$ (this operation is meaningful of course only if $z_{c}>1$ ). $\phi_{c}(z)$ is a new pgf because $\phi_{c}(1)=z_{c}^{-1} \phi\left(z_{c}\right) \leq 1$. We have $\pi_{c}(k)=\left[z^{k}\right] \phi_{c}(z)=$ $z_{c}^{k-1} \pi(k)$, so one can work with $\phi_{c}$ as well. We also have $\phi_{c}(z)=f \circ g(z)$ with $g(z)=1-(1-z)^{-\theta}$ and $f(z)=1-\left(a+b z_{c}^{\theta}-a z\right)^{-1 / \theta}$. This allows to compute $\pi(k)$ by Faa di Bruno formula for the composition of Taylor series. First we have $\pi(0)=z_{c}\left(1-\left(a+b z_{c}^{\theta}\right)^{-1 / \theta}\right)$. By Faa di Bruno formula ([4], Tome 1, p. 149), then

\section{Proposition 11}

$$
\pi(k)=\frac{1}{k ! z_{c}^{k-1}} \sum_{l=1}^{k} f_{l} B_{k, l}\left(g_{\bullet}\right), k \geq 1,
$$

where $f_{l}$ are the Taylor coefficients of $f(z)$ and $B_{k, l}\left(g_{\bullet}\right)$ the Bell polynomials in the indeterminate $g_{\bullet}:=\left(g_{1}, g_{2}, \ldots\right)$, the $g_{k} s$ being the Taylor coefficients of $g(z)$. The Bell polynomials are defined by

$$
B_{k, l}\left(g_{\bullet}\right)=\frac{k !}{l !}\left[z^{k}\right] g(z)^{l}
$$

with the boundary conditions

$$
\begin{gathered}
B_{k, 0}\left(g_{\bullet}\right)=B_{0, l}\left(g_{\bullet}\right)=0, k, l \geq 1 \text { and } B_{0,0}\left(g_{\bullet}\right):=1, \text { and }, \\
B_{k, 1}\left(g_{\bullet}\right)=g_{k} \text { and } B_{k, k}\left(g_{\bullet}\right)=g_{1}^{k} .
\end{gathered}
$$

This computation of $\pi(k)$ is in agreement with Proposition 4 of Sagitov and Lindo but our representation and its proof, inspired from Faa di Bruno formulae and making use of Bell polynomials, are different. We now list some properties concerning the coefficients $f_{l}$ and $B_{k, l}\left(g_{\bullet}\right)$. We first recall that 4],

$$
B_{k, l}\left(x_{\bullet}\right)=k ! \sum^{*} \prod_{j \geq 1} \frac{1}{c_{j} !}\left(\frac{x_{j}}{j !}\right)^{c_{j}},
$$

the latter star summations running over the integers $c_{j}$ obeying $\sum_{j \geq 1} c_{j}=l$ and $\sum_{j>1} j c_{j}=k \geq l$.

We note now that, with $[a]_{l}=a(a+1) \ldots(a+l-1)$ the ascending factorial with $[a]_{0}:=1, C=\left(a+b z_{c}^{\theta}\right)^{-1 / \theta}$ and $D=\left(a+b z_{c}^{\theta}\right) / a=C^{-\theta} / a, f(z)=1-$ $C(1-z / D)^{-1 / \theta}$ with Taylor coefficients

$$
f_{0}=1-C \text { and } f_{l}=-\frac{C}{\theta}[1+1 / \theta]_{l-1} D^{-l}=-C D^{-l}[1 / \theta]_{l}, l \geq 1 .
$$


For the case $g(z)=1-(1-z)^{-\theta}$, it holds $g_{\bullet}=-\theta[1+\theta]_{\bullet-1}=-[\theta]_{\bullet}$. Because $g_{1}=-\theta$ and $g_{m+1}=g_{m}(m+\theta), m \geq 1$, it follows that the Bell coefficients $B_{k, l}\left(g_{\bullet}\right)$ for this function $g$ obey a simple 3 -term recursion

$$
B_{k+1, l}\left(g_{\bullet}\right)=-\theta B_{k, l-1}\left(g_{\bullet}\right)+(k+l \theta) B_{k, l}\left(g_{\bullet}\right), k, l \geq 1 .
$$

For instance $B_{1,1}\left(g_{\bullet}\right)=-\theta$ leading to $\pi(1)=f_{1} B_{1,1}\left(g_{\bullet}\right)=C D^{-1}, B_{2,1}\left(g_{\bullet}\right)=$ $(1+\theta) B_{1,1}\left(g_{\bullet}\right)=-\theta(1+\theta)$, leading to $\pi(2)=\frac{1}{2 z_{c}}\left(f_{1} B_{2,1}\left(g_{\bullet}\right)+f_{2} B_{2,2}\left(g_{\bullet}\right)\right)=$ $\frac{1}{2 z_{c}}(1+\theta) C D^{-2}(D-1), \ldots$ The formulae (21), (22) and (23) completely characterize the $\pi(k)$ s. The $B_{k, l}\left(g_{\bullet}\right)$ constitute generalized Stirling numbers studied in [3].

Remark: If $\theta=-1 / L$ where $L>1$ is an integer, $f(z)=1-C(1-z / D)^{L}$ is a polynomial of degree $L$ in $z$ so $f_{l}=0$ if $l>L$ which largely simplifies (21). Furthermore, in this case, $g_{k}=L^{-k} \prod_{l=1}^{k-1}(l L-1)$. If $L=2, g_{k}=2^{-2(k-1)}(2 k-3) ! /(k-2)$ !.

- The transition matrix and its powers. We now first wish to compute $P_{a, b}(i, j)=\left[z^{j}\right] \phi(z)^{i}=z_{c}^{i-j}\left[z^{j}\right] \phi_{c}(z)^{i}$, the transition matrix of the $\theta$-branching process, where its dependence on the parameters $(a, b)$ has been emphasized. We have $\phi_{c}(z)^{i}=f_{i} \circ g(z)$, still with $g(z)=1-(1-z)^{-\theta}$ and now with $f_{i}(z):=$ $\left[1-\left(a+b z_{c}^{\theta}-a z\right)^{-1 / \theta}\right]^{i}$. So with $f_{i, k}, k \geq 1$, the Taylor coefficients of $f_{i}(z)$, we similarly get

\section{Proposition 12}

$$
P_{a, b}(i, j)=\frac{z_{c}^{i-j}}{j !} \sum_{k=1}^{j} f_{i, k} B_{j, k}\left(g_{\bullet}\right) .
$$

We note that $f_{i}(z)=h_{i}(\mathrm{f}(z))$ where $h_{i}(z)=(1-C+C z)^{i}$ and $\mathrm{f}(z):=1-$ $(1-z / D)^{-1 / \theta}$ so that with $h_{i, l}=\frac{i !}{(i-l) !}(1-C)^{i-l} C^{l}(=0$ if $l>i)$ and with $\mathrm{f}_{\bullet}$ given from (22) as $\mathrm{f}_{l}=-[1 / \theta]_{l} D^{-l}, l \geq 1$, by Faa di Bruno formula again

$$
f_{i, 0}=(1-C)^{i} \text { and } f_{i, k}=\sum_{l=1}^{k \wedge i} h_{i, l} B_{k, l}\left(\mathrm{f}_{\bullet}\right) .
$$

Note $\pi(j)=P_{a, b}(1, j)$ as required.

To obtain now $P_{a, b}^{n}(i, j)$, the $(i, j)$-entry of the $n$-th power of $P_{a, b}$, we just need to substitute $\left(a_{n}=a^{n}, b_{n}=b\left(1+a+\ldots+a^{n-1}\right)\right)$ to $(a, b)$, so it simply holds

$$
P_{a, b}^{n}(i, j)=P_{a_{n}, b_{n}}(i, j),
$$

taking advantage of the invariance under iteration of the $\theta$-family when $\theta \in(-1,1) \backslash\{0\}$. We note that the dependence on $n$ in $P_{a_{n}, b_{n}}(i, j)$ is only in the coefficients $f_{i, k}$ in (24), through $C$ and $D$. To emphasize this point, we shall also write

Corollary 13

$$
P_{a, b}^{n}(i, j)=P_{a_{n}, b_{n}}(i, j)=\frac{z_{c}^{i-j}}{j !} \sum_{k=1}^{j} f_{i, k}^{(n)} B_{j, k}(g \bullet)
$$

where $f_{i, k}^{(n)}$ is obtained from $f_{i, k}$ in (25) while substituting $\left(a_{n}=a^{n}, b_{n}=b\left(1+a+\ldots+a^{n-1}\right)\right)$ to $(a, b)$ in the expressions of $C=\left(a+b z_{c}^{\theta}\right)^{-1 / \theta}$ and $D=\left(a+b z_{c}^{\theta}\right) / a$.

It remains to discuss the special integral cases for $\theta$. 


\subsection{The case $\theta=0$}

We recall that $\phi(z)=z_{c}-\lambda\left(z_{c}-z\right)^{a}$, where $\lambda=\left(z_{c}-\rho\right)^{1-a}$ and $\rho$ obeys $\phi(\rho)=\rho$. With $\phi_{c}(z)=1-\lambda z_{c}^{a-1}(1-z)^{a}$ and $\pi_{c}(k)=\left[z^{k}\right] \phi_{c}(z), \pi(k)=\pi_{c}(k) / z_{c}^{k-1}$ with $\pi_{c}(k)=-\lambda z_{c}^{a-1}[-a]_{k} / k$ !. Next, with $\lambda_{c}:=\lambda z_{c}^{a-1}$

$$
\phi_{c}(z)^{i}=\left(1-\lambda_{c}(1-z)^{a}\right)^{i}=\left(1-\lambda_{c}+\lambda_{c}\left(1-(1-z)^{a}\right)\right)^{i}=h_{i} \circ g(z),
$$

with $g(z)=1-(1-z)^{a}$ and $h_{i}(z)=\left(1-\lambda_{c}+\lambda_{c} z\right)^{i}$. With $g_{\bullet}=-[-a]_{\bullet}$ and $h_{i, k}=\frac{i !}{(i-k) !}\left(1-\lambda_{c}\right)^{i-k} \lambda_{c}^{k}$, we thus get similarly

$$
P_{a, \lambda}(i, j)=\frac{z_{c}^{i-j}}{j !} \sum_{k=1}^{j} h_{i, k} B_{j, k}\left(g_{\bullet}\right) \text { and } P_{a, \lambda}^{n}(i, j)=P_{a_{n}, \lambda_{n}}(i, j),
$$

where $a_{n}=a^{n}(a \in(0,1))$ and $\lambda_{n}=\lambda^{\left(1-a^{n}\right) /(1-a)}$. The $B_{j, k}\left(g_{\bullet}\right)$ also obey a three terms recursion of the type (23) with $-a$ substituted to $\theta$. Note $\pi(j)=P_{a, \lambda}(1, j)$ as required.

\subsection{The case $\theta=1$}

With $\phi(z)=z_{c}-\left(a\left(z_{c}-z\right)^{-1}+b\right)^{-1}$ we wish to compute $\pi(k)=\left[z^{k}\right] \phi(z)$ with $\pi(0)=z_{c}(a+b-1) /(a+b)$ in the first place. Introduce $\phi_{c}(z)=z_{c}^{-1} \phi\left(z_{c} z\right)$, so with $\phi_{c}(z)=1-\left(a(1-z)^{-1}+b z_{c}\right)^{-1}$. We have $\phi_{c}(z)=f \circ g(z)$ with $g(z):=$ $(1-z)^{-1}-1$ and $f(z)=1-\left(a+b z_{c}+a z\right)^{-1}=1-C(1+z / D)^{-1}$ where $C=$ $\left(a+b z_{c}\right)^{-1}$ and $D=\left(a+b z_{c}\right) / a$. Let $f_{l}$ be the Taylor coefficients of $f(z)$ and $g_{k}$ the Taylor coefficients of $g(z)$. By Faa di Bruno formula

$$
\pi(k)=\frac{1}{k ! z_{c}^{k-1}} \sum_{l=1}^{k} f_{l} B_{k, l}\left(g_{\bullet}\right), k \geq 1,
$$

with $g_{k}=k$ ! and $f_{0}=1-C$ and $f_{l}=(-1)^{l-1} C D^{-l} l !, l \geq 1$. We have $B_{k, l}(\bullet !)=$ $\left(\begin{array}{c}k-1 \\ l-1\end{array}\right) \frac{k !}{l !}$, so

$$
\pi(k)=\frac{C}{z_{c}^{k-1}} \sum_{l=1}^{k}\left(\begin{array}{c}
k-1 \\
l-1
\end{array}\right)(-1)^{l-1} D^{-l}=C D^{-1}\left(\frac{1-D^{-1}}{z_{c}}\right)^{k-1}, k \geq 1 .
$$

Next,

$$
P_{a, b}(i, j)=z_{c}^{i-j}\left[z^{j}\right] \phi_{c}(z)^{i} .
$$

We have $\phi_{c}(z)^{i}=f_{i} \circ g(z)$ still with $g(z)=(1-z)^{-1}-1$ and now with $f_{i}(z)=$ $\left[1-C(1+z / D)^{-1}\right]^{i}$. So with $f_{i, k}, k \geq 1$, the Taylor coefficients of $f_{i}(z)$ and with $B_{j, k}(\bullet !)=\left(\begin{array}{c}j-1 \\ k-1\end{array}\right) \frac{j !}{k !}$, we get similarly

$$
P_{a, b}(i, j)=\frac{z_{c}^{i-j}}{j !} \sum_{k=1}^{j} f_{i, k} B_{j, k}\left(g_{\bullet}\right)
$$

It remains to compute the $f_{i, k} \mathrm{~s}$. We note that $f_{i}(z)=h_{i}(f(z))$ where $h_{i}(z)=$ $(1-C-C z)^{i}$ and $f(z)=(1+z / D)^{-1}-1$ so that with $h_{i, l}=\frac{i !}{(i-l) !}(1-C)^{i-l}(-C)^{l}$ 
and with $f_{\bullet}$ given by $f_{l}=(-D)^{-l} l !, l \geq 1$, by Faa di Bruno formula again

$$
f_{i, 0}=(1-C)^{i} \text { and } f_{i, k}=\sum_{l=1}^{k \wedge i} h_{i, l} B_{k, l}\left(f_{\bullet}\right) .
$$

Now, $B_{k, l}\left(f_{\bullet}\right)=(-D)^{-k} B_{k, l}(\bullet !)=(-D)^{-k}\left(\begin{array}{c}k-1 \\ l-1\end{array}\right) \frac{k !}{l !}$ and

$$
f_{i, k}=k !(1-C)^{i} D^{-k} \sum_{l=1}^{k \wedge i}(-1)^{k-l}\left(\begin{array}{l}
i \\
l
\end{array}\right)\left(\begin{array}{l}
k-1 \\
l-1
\end{array}\right)\left(\frac{C}{1-C}\right)^{l} .
$$

Exchanging the summation over $k$ and $l$ in (30) and applying the binomial identity (keeping in mind $D^{-1}=a C$ )

$$
P_{a, b}(i, j)=z_{c}^{i-j}(1-C)^{i}\left(1-D^{-1}\right)^{j} \sum_{l=1}^{i \wedge j}\left(\begin{array}{l}
i \\
l
\end{array}\right)\left(\begin{array}{l}
j-1 \\
l-1
\end{array}\right)\left(\frac{C}{1-C} \frac{D^{-1}}{1-D^{-1}}\right)^{l} .
$$

To obtain now $P_{a, b}^{n}(i, j)$, the $(i, j)$-entry of the $n$-th power of $P_{a, b}$, we just need to substitute $\left(a_{n}=a^{n}, b_{n}=b\left(1+a+\ldots+a^{n-1}\right)\right)$ to $(a, b)$ in $(C, D)$, so it simply holds

$$
P_{a, b}^{n}(i, j)=P_{a_{n}, b_{n}}(i, j),
$$

where $P_{a, b}(i, j)$ is given by (32). The resulting expression generalizes Proposition 2.2 of [1].

\subsection{The case $\theta=-1$ (Greenwood model)}

Here $\phi(z)=a z+z_{c}(1-a)-b$. We get $\pi(1)=a, \pi(0)=z_{c}(1-a)-b \leq 1-\pi(1)$. We have

$$
P(i, j)=\left[z^{j}\right] \phi(z)^{i}=\left(\begin{array}{l}
i \\
j
\end{array}\right) \pi(0)^{i-j} \pi(1)^{j},
$$

and

$$
P^{n}(i, j)=\left[z^{j}\right] \phi^{\circ n}(z)^{i}=\left(\begin{array}{c}
i \\
j
\end{array}\right) \pi_{n}(0)^{i-j} \pi_{n}(1)^{j},
$$

where $\pi_{n}(1)=\pi(1)^{n}$ and $\pi_{n}(0)=\pi(0) \frac{1-\pi(1)^{n}}{1-\pi(1)}$. Both $P$ and $P^{n}$ have binomial entries with $P^{n}(i, i)=\pi(1)^{n i}$. If $\pi(0)+\pi(1)=1$ (the regular case), $\pi_{n}(0)+\pi_{n}(1)=$ 1 and $P^{n}$ is stochastic. If $\pi(0)+\pi(1)<1$ (the explosive case), $\pi_{n}(0)+\pi_{n}(1)<1$ and $P^{n}$ is sub-stochastic. To make it stochastic, we can add state $\{\infty\}$ to the statespace and assume that it is absorbing. We can thus complete $P$ to make it stochastic while considering $P(i, \infty)=1-\sum_{j=0}^{i}\left(\begin{array}{l}i \\ j\end{array}\right) \pi(0)^{i-j} \pi(1)^{j}=1-(\pi(0)+\pi(1))^{i}$ and $P(\infty, \infty)=1$. If $\phi(1)=1$, such regular pure death process was recently considered by [13, revisiting the Greenwood model of infectiousness, [6].

\subsection{Resolvent of the $\theta$-linear fractional processes}

With $\delta_{i, j}$ the Kronecker delta, for $i, j \geq 1$, we also obtain the resolvent of $N_{n}(i)$ as

$$
g_{i, j}(z):=\delta_{i, j}+\sum_{n \geq 1} z^{n} P^{n}(i, j) .
$$

In particular,

$$
g_{i, i}(z)=1+\sum_{n \geq 1} z^{n} P^{n}(i, i) .
$$


Note $g_{i, j}(1)=\delta_{i, j}+\mathbf{E}\left(\sum_{n \geq 1} \mathbf{1}_{\left\{N_{n}(i)=j\right\}}\right)$, the expected value of the time spent on state $j$ starting from $i$, is the Green kernel.

Proposition 14 Using (27), with $F_{i, k}(z):=\sum_{n \geq 1} z^{n} f_{i, k}^{(n)}$, we get the following tricky expression for the resolvent

$$
g_{i, j}(z):=\delta_{i, j}+\sum_{n \geq 1} z^{n} P_{a_{n}, b_{n}}(i, j)=\delta_{i, j}+\frac{z_{c}^{i-j}}{j !} \sum_{k=1}^{j} F_{i, k}(z) B_{j, k}\left(g_{\bullet}\right) .
$$

These quantities are fundamental to compute pgfs of important quantities such as passage times. It holds for example that $\mathbf{E}\left(z^{\tau_{i, j}}\right)=g_{i, j}(z) / g_{j, j}(z)$ where

$$
\tau_{i, j}=\inf \left(n \geq 1: N_{n}(i)=j\right)
$$

is the first passage time to state $j \neq i$ of $N_{n}$ given $N_{0}=i$, 14, [19. In particular $\mathbf{P}\left(\tau_{i, j}<\infty\right)=g_{i, j}(1) / g_{j, j}(1)$ are the hitting probabilities of state $j$ starting from $i$. Furthermore, with

$$
\tau_{i, i}^{*}=\inf \left(n \geq 1: N_{n}(i)=i\right),
$$

the first return time to state $i$ of $N_{n}(i)$, it holds by renewal arguments that $\mathbf{E}\left(z^{\tau_{i, i}^{*}}\right)=$ $1-1 / g_{i, i}(z)$, 14]. In particular $\mathbf{P}\left(\tau_{i, i}^{*}<\infty\right)=1-1 / g_{i, i}(1)$. Therefore for example, the mean return time to state $i$ given $\tau_{i, i}^{*}<\infty$ is

$$
\mathbf{E}\left(\tau_{i, i}^{*} \mid \tau_{i, i}^{*}<\infty\right)=\frac{g_{i, i}^{\prime}(1)}{g_{i, i}(1)\left(g_{i, i}(1)-1\right)},
$$

whenever this quantity exists.

Let us briefly sketch what this says for the simplest Greenwood model example when $\theta=-1$ : firstly $P^{n}(i, i)=\pi_{n}(1)^{j}$ leading to $g_{i, i}(z)=1+\sum_{n \geq 1} z^{n} \pi(1)^{n i}=$ $1 /\left(1-z \pi(1)^{i}\right)$. Therefore $\mathbf{E}\left(z^{\tau_{i, i}^{*}}\right)=z \pi(1)^{i}$, translating the fact that $\tau_{i, i}^{*}=1$ with probability $\pi(1)^{i},=\infty$ with probability $1-\pi(1)^{i}$ (the no return to $i$ event if in the first step one of the $i$ founders moved to one of the absorbing states, 0 or $\infty$ ). In addition, in the regular case $\pi(0)=1-\pi(1)$,

$\mathbf{P}\left(\tau_{i, j}<\infty\right)=g_{i, j}(1) / g_{j, j}(1)=\left(1-\pi(1)^{j}\right)\left(1+\sum_{n \geq 1}\left(\begin{array}{l}i \\ j\end{array}\right)\left(1-\pi(1)^{n}\right)^{i-j} \pi(1)^{n j}\right)$,

which, upon developing $\left(1-\pi(1)^{n}\right)^{i-j}$ and summing over $n$ is Proposition 1.1 and Theorem 1.2 of [13].

\section{One illustrative example}

As an illustrative application of the previous results, let us look for the value of $n$ for which a supercritical process as in $(C)$ will nearly never (with large probability c) go extinct as soon as $N_{n}(i)>0$. It is given by (6)

$$
c=\frac{1-\rho^{i}}{1-\phi^{o n}(0)^{i}}=: 1-\epsilon
$$


When $\epsilon$ is small, it leads to

$$
\rho-\phi^{\circ n}(0) \approx \epsilon \cdot \frac{1-\rho^{i}}{i \rho^{i-1}} .
$$

The condition for this Taylor expansion to be valid is given 2 by $\rho-\phi^{\circ n}(0) \ll \rho /(i-1)$ or alternatively

$$
\epsilon \ll \frac{\rho^{i}}{1-\rho^{i}} .
$$

The relation (38) shows that for small $\epsilon$, having $i$ founders amounts simply to multiply $\epsilon$ by a factor that only depends on the value of the fixed point $\rho$ and the number $i$. Let us now use the explicit form of the supercritical $\theta$-linear fractional pgfs. In that case, it holds that

$$
a^{n}=\frac{\left(z_{c}-\phi^{\circ n}(z)\right)^{-\theta}-\left(z_{c}-\rho\right)^{-\theta}}{\left(z_{c}-z\right)^{-\theta}-\left(z_{c}-\rho\right)^{-\theta}} .
$$

A Taylor expansion of $\left(z_{c}-\phi^{o n}(z)\right)^{-\theta}$ for small $\rho-\phi^{\circ n}(0)$ yields

$$
-\theta \frac{\rho-\phi^{\circ n}(z)}{\rho-z_{c}} \approx a^{n}\left(1-\left(\frac{z_{c}-z}{z_{c}-\rho}\right)^{-\theta}\right),
$$

the Taylor expansion validity condition being $\left|\rho-\phi^{\circ n}(z)\right| \ll\left|\left(z_{c}-\rho\right) / \theta\right|$. Combined with the previous result, we get

$$
a^{n} \approx \epsilon \cdot \frac{1-\rho^{i}}{i \rho^{i}} \cdot(-\theta) \frac{1-\alpha}{1-\alpha^{-\theta}},
$$

with $\alpha:=z_{c} /\left(z_{c}-\rho\right)>1$; the validity conditions are

$$
\epsilon \ll \frac{\rho^{i}}{1-\rho^{i}} \text { and } \epsilon \ll \frac{i \rho^{i}}{|\theta|(\alpha-1)} .
$$

This shows that the searched value of $n$ for which a supercritical process as in $(C)$ will nearly never go extinct is approximately the sum of three terms:

- one, related to the required accuracy, that is the logarithm of $\epsilon$ in base $a$. In particular, to have a result 10 times more precise, one has to wait $\left|\log _{a}(10)\right|$ more steps,

- one, related to the number $i$ of founders, which also depends on the parameters $a$ and $\rho$,

- one, related to the model parameters only, which depends on $a, \theta$, and $z_{c}$ and $\rho$ through $\alpha$.

For instance, taking $a=0.63$ (so that $a^{5} \approx 0.1$ ), $\rho=0.7, z_{c}=1$, we get:

- when $\theta=+1$ and $i=1,9$ generations are needed if the population is to survive with a probability $1-10^{-2} .5$ more generations will increase this probability to $1-10^{-3}$, and another 5 to $1-10^{-4}$.

\footnotetext{
${ }^{2}$ It is assumed here that $i>1$. If $i=1$, the condition on $\rho-\phi^{\circ n}(0)$ is no longer valid, but the one on $\epsilon$ still is.
} 
- when $\theta=+1$, with an uncertainty $\epsilon=10^{-4}$ and eight founders, the time to wait decreases to 16 generations. With thirteen founders, it decreases further to 13 generations. Notice that $\rho^{19} \approx 0.001$, so one has to be careful not to get out of the range of (39).

- with one founder and an uncertainty $10^{-4}, 19$ generations are needed for $\theta=1$, 20 generations for the limit $\theta=0$ and 21 for $\theta=-1$.

In all these special cases, we conclude that if extinction is to occur, it occurs rapidly or nearly never.

\section{Acknowledgments:}

T. Huillet acknowledges partial support from the "Chaire Modélisation mathématique et biodiversité". N. Grosjean and T. Huillet also acknowledge support from the labex MME-DII Center of Excellence (Modèles mathématiques et économiques de la dynamique, de l'incertitude et des interactions, ANR-11-LABX-0023-01 project).

\section{References}

[1] Athreya, K. B. and Ney, P. Branching Processes. Springer, New York, 1972.

[2] Biggins, J. D.; Shanbhag, D. N. Some divisibility problems in branching processes. Math. Proc. Cambridge Philos. Soc. 90, no. 2, 321-330, (1981).

[3] Charalambides, Ch. A.; Singh, J. A review of the Stirling numbers, their generalizations and statistical applications. Comm. Statist. Theory Methods, 17, no. 8 (1988).

[4] Comtet, L. Analyse combinatoire. Tomes 1 et 2. Presses Universitaires de France, Paris, 1970.

[5] Feller W. An introduction to probability theory and its applications, 2, Wiley, New York, 1971.

[6] Greenwood, M. On the statistical measure of infectiousness. J. Hyg. Camb. 31, 336-351, (1931).

[7] Harris, T. E. The theory of branching processes. Die Grundlehren der Mathematischen Wissenschaften, Bd. 119 Springer-Verlag, Berlin; Prentice-Hall, Inc., Englewood Cliffs, N.J. 1963.

[8] Hénard, O. The fixation line in the Lambda-coalescent. Ann. Appl. Prob. Volume 25, Number 5, 3007-3032, (2015).

[9] Hoppe, F. M. On a Schröder equation arising in branching processes. Aequationes Mathematicae, 20(1), 33-37, (1980).

[10] Huillet, T. On Mittag-Leffler distributions and related stochastic processes. J. of Comp. and Appl. Math., Volume 296, Pages 181-211, (2016).

[11] Klebaner, F. C.; Rösler, U. and Sagitov, S. Transformations of Galton-Watson processes and linear fractional reproduction. Adv. in Appl. Probab. Volume 39, Number 4, 1036-1053, (2007).

[12] Lambert, A. Some aspects of discrete branching processes. http://www.cmi.univ-mrs.fr/ pardoux/Ecole_CIMPA/CoursALambert.pdf, (2010).

[13] Möhle, M. On hitting probabilities for the Greenwood model. Preprint March 2016.

[14] Norris, J. R. Markov chains. Cambridge University Press, 1998.

[15] Rogers, L. C. G.; Williams, D. Diffusions, Markov processes and Martingales. Vol 1, Foundations, 2nd edition, John Wiley, Chichester, (1994). 
[16] Sagitov S.; Lindo A. A special family of Galton-Watson processes with explosions. In Branching Processes and Their Applications. Lect. Notes Stat. Proc. (I.M. del Puerto et al eds.) Springer, Berlin, 2016 (to appear). arxiv.org/pdf/1502.07538, (2015).

[17] Schröder, E. Über iterierte funktionen. Math, Ann. 3, 296-322, (1871).

[18] Steutel, F. W.; van Harn, K. Discrete analogues of self-decomposability and stability. Ann. Prob., 7, 893-899, (1979).

[19] Woess, W. (2009). Denumerable Markov chains. Generating functions, boundary theory, random walks on trees. EMS Textbooks in Mathematics. European Mathematical Society (EMS), Zürich.

[20] Yaglom, A. M. Certain limit theorems of the theory of branching stochastic processes. Doklady Akademii Nauk SSSR, 56, 795-798, (1947). 